\title{
Article \\ Perioperative Predictors of Early Recurrence for Resectable and Borderline-Resectable Pancreatic Cancer
}

\author{
Masafumi Imamura *(D), Minoru Nagayama (D), Daisuke Kyuno (D), Shigenori Ota (D), Takeshi Murakami,

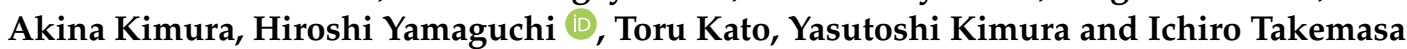

check for

updates

Citation: Imamura, M.; Nagayama, M.; Kyuno, D.; Ota, S.; Murakami, T.; Kimura, A.; Yamaguchi, H.; Kato, T.; Kimura, Y.; Takemasa, I. Perioperative Predictors of Early Recurrence for Resectable and Borderline-Resectable Pancreatic Cancer. Cancers 2021, 13, 2285. https://doi.org/10.3390/ cancers13102285

Academic Editor: Tsutomu Fujii

Received: 1 April 2021

Accepted: 6 May 2021

Published: 11 May 2021

Publisher's Note: MDPI stays neutral with regard to jurisdictional claims in published maps and institutional affiliations.

Copyright: (c) 2021 by the authors. Licensee MDPI, Basel, Switzerland. This article is an open access article distributed under the terms and conditions of the Creative Commons Attribution (CC BY) license (https:// creativecommons.org/licenses/by/ $4.0 /)$.
Department of Surgery, Surgical Oncology and Science, Sapporo Medical University, Sapporo 060-8543, Japan; mnagayam@sapmed.ac.jp (M.N.); kyuno@sapmed.ac.jp (D.K.); oshige@sapmed.ac.jp (S.O.); murakami.takeshi@sapmed.ac.jp (T.M.); akina030@sapmed.ac.jp (A.K.); hyama@sapmed.ac.jp (H.Y.); toru.kato1969@gmail.com (T.K.); kimuray@sapmed.ac.jp (Y.K.); itakemasa@sapmed.ac.jp (I.T.)

* Correspondence: imamura@sapmed.ac.jp; Tel.: +81-11-011-611-2111 (ext. 32810)

Simple Summary: Most patients with a pancreatic ductal adenocarcinoma develop a recurrence after surgery. Predictive factors may therefore guide therapeutic decision-making. We aimed to identify perioperative predictors of the early recurrence of pancreatic ductal adenocarcinomas. We found that preoperative $(>52 \mathrm{U} / \mathrm{mL})$ and postoperative $(>37 \mathrm{U} / \mathrm{mL}$ ) elevated carbohydrate antigen 19-9 levels as well as a tumor size $>3.0 \mathrm{~cm}$ were independently associated with an early recurrence after a pancreatectomy. Furthermore, an early recurrence resulted in a more frequent liver metastasis than a late recurrence, suggesting that patients experiencing a recurrence within 12 months had undetectable micrometastases. Further studies are needed to identify new biomarkers for the detection of clinically occult micrometastases during surgery as current preoperative risk factors are inadequate to accurately identify patients susceptible to an early recurrence of pancreatic ductal adenocarcinomas.

Abstract: We aimed to identify the perioperative predictors of the early recurrence (ER) of resectable and borderline-resectable pancreatic ductal adenocarcinomas (PDACs). After surgery for a PDAC, most patients develop a recurrence. Predictive factors may therefore guide therapeutic decisionmaking. Patients $(n=234)$ who underwent a pancreatectomy for a PDAC between 2006 and 2019 were included. The postrecurrence survival (PRS) was estimated using Kaplan-Meier curves. Predictive factors for an ER were assessed using logistic regression analyses; 93 patients (39.7\%) were recurrencefree at the last follow-up. Patients with an ER $(n=85,36.3 \%)$, defined as a recurrence within the first 12 months after surgery, had 1- and 2-year PRS rates of 38.7\% and 9.5\%, respectively, compared with $66.9 \%$ and $37.2 \%$ for those with a late recurrence $(n=56,23.9 \%$; both $p<0.001)$. The most common site of an ER was the liver (55.3\%) with a significantly shorter median overall survival time than that with either a local or a lung recurrence (14.5 months; $p<0.001)$. Preoperative and postoperative risk factors for an ER included a tumor size $>3.0 \mathrm{~cm}$ (odds ratio (OR): 3.11, 95\% confidence interval (CI): 1.35-7.14) and preoperative carbohydrate antigen 19-9 (CA19-9) levels >52 U/mL (OR: 3.25, 95\% CI: 1.67-6.30) and a pathological tumor size $>3.0 \mathrm{~cm}$ (OR: 2.00, 95\% CI: 1.03-3.90) and postoperative carbohydrate antigen 19-9 levels $>37 \mathrm{U} / \mathrm{mL}$ (OR: 2.11,95\% CI: 1.02-4.36), respectively. Preoperatively $(>52 \mathrm{U} / \mathrm{mL})$ and postoperatively $(>37 \mathrm{U} / \mathrm{mL}$ ) elevated CA19-9 and a tumor size $>3.0 \mathrm{~cm}$ were independent predictors for an ER after a pancreatectomy for a PDAC.

Keywords: carbohydrate antigen 19-9; early recurrence; pancreatic ductal adenocarcinoma; postrecurrence survival

\section{Introduction}

A pancreatic ductal adenocarcinoma (PDAC), one of the most aggressive cancers worldwide, is predicted to become the second leading cause of cancer-related deaths in Western countries within the next 10 years [1]. In Japan, a PDAC is the fourth leading 
cause of cancer-related deaths; the number of patients is predicted to increase in the future [2]. A complete tumor resection remains the only potentially curative option for a PDAC. The criteria for resectability have been proposed by the National Comprehensive Cancer Network (NCCN) [3]. Recommended therapeutic strategies are applied according to this classification to improve prognosis; however, even in cases classified as resectable according to the NCCN guidelines, a low postoperative survival rate has been reported [4,5]. Moreover, approximately $80 \%$ of patients experience local and metastatic recurrence, with $>50 \%$ occurring within the first 12 months after curative surgery [6].

Recurrence within the first 12 months after surgery is considered to be an "early recurrence" (ER) and is a characteristic of a PDAC. The perioperative predictors of an ER such as the tumor size [6-8], metastases in the harvested lymph nodes [6-8], the serum carbohydrate antigen 19-9 (CA19-9) value [6,9-12], the duration of symptoms [13], a modified Glasgow Prognostic Score [14], a Charlson age-comorbidity index [6], tumor differentiation $[6,12,13]$ and p53 expression in the primary tumor [15] have been reported to identify high-risk patients. Recently, neoadjuvant therapy has been shown to provide oncological benefits more than upfront surgery in patients with a borderline-resectable PDAC (BR-PDAC) [16,17]; significant survival benefits of neoadjuvant chemotherapy have also been demonstrated in phase III of the study on patients with a resectable PDAC (R-PDAC) [18].

Currently, more PDAC patients are being treated with neoadjuvant therapy prior to curative surgery; however, most of the above reports on perioperative predictors were studies involving an R-PDAC. These studies do not mention the association between resectability or neoadjuvant therapy and an ER; therefore, it is necessary to identify risk factors for an ER while taking these into consideration. Potential predictive factors for a postoperative ER may guide decision-making to extend the neoadjuvant therapy duration as well as the choice of adjuvant therapy.

The purpose of this study was to identify perioperative predictors of an ER of an R- and BR-PDAC. We focused on the time of recurrence, patterns of recurrence, postrecurrence survival (PRS) and perioperative clinicopathological factors for an ER after a curative resection.

\section{Materials and Methods}

\subsection{Study Population and Inclusion and Exclusion Criteria}

Between January 2006 and December 2019, 265 consecutive patients underwent a pancreatic resection for a pancreatic adenocarcinoma at Sapporo Medical University Hospital (Sapporo, Japan). All patients were histologically diagnosed with a ductal adenocarcinoma of the pancreas confirmed by two pathologists. The exclusion criteria were as follows: an intraductal papillary mucinous carcinoma or a pancreatic intraepithelial neoplasia, incomplete records owing to follow-up at other institutions or $<12$ months of follow-up. Of the 265 patients, 31 were excluded owing to conversion surgery for an unresectable cancer $(n=23)$, a re-pancreatectomy for recurrence in the remnant pancreas after an initial pancreatectomy $(n=6)$ and 30-day postoperative mortality $(n=2)$. Data of the remaining 234 patients were retrospectively analyzed. All 234 patients included in this study were preoperatively diagnosed with an R-or BR-PDAC according to the NCCN guidelines (version 1, 2019) [3] during a multidisciplinary team meeting.

\subsection{Outcome Measures}

Data on preoperative and postoperative demographics as well as clinicopathological and treatment characteristics were extracted from a prospectively maintained institutional database. Preoperative and postoperative CA19-9 levels were obtained, when available. CA19-9 measurements were performed within one month before surgery; CA19-9 levels acquired at a time of jaundice (total bilirubin $>5 \mathrm{mg} / \mathrm{dL}$ ) or later than two months postoperatively were excluded from the analysis. Furthermore, CA19-9 levels $<3 \mathrm{U} / \mathrm{mL}$ were related to Lewis-negative patients [19] and were also excluded from the analysis. 
The preoperative tumor diameter was measured using endoscopic ultrasonography both at the first examination and prior to neoadjuvant therapy [20]. The surgical procedure involved a standard or subtotal stomach- or pylorus-preserving pancreatoduodenectomy in $155(66.3 \%)$, a distal pancreatectomy in $74(31.6 \%)$ and a total pancreatectomy in 5 $(2.1 \%)$ patients. A regional lymph node dissection was performed on all patients; resected specimens were fixed in $10 \%$ formalin at room temperature. The size and gross appearance of the tumor were recorded. The pathological stage of all tumor specimens was determined according to the Union for International Cancer Control Tumor-Node-Metastasis (TNM) staging system (eighth edition) [21]. The tumor status following surgery was defined using the residual tumor (R) classification: R0, no residual tumor; R1, a microscopic residual tumor; R2, a macroscopic residual tumor.

\subsection{Neoadjuvant/Adjuvant Therapy and Follow-Up}

Neoadjuvant therapy has been administered to R- and BR-PDAC patients since 2008 exclusively when the patients voluntarily registered for the clinical trial at the time and their respective attending physicians obtained informed consent from them. Neoadjuvant therapy involved chemoradiotherapy in $25(10.7 \%)$, nab-paclitaxel plus gemcitabine in 20 $(8.5 \%)$ and S-1 in $9(3.8 \%)$ patients. Adjuvant therapy has been administered postoperatively to most patients since 2006. Adjuvant therapy involved gemcitabine according to the results of the CONKO-001 trial [22]. S-1 has been administered since 2013 according to the results of the JASPAC01 trial [23].

The patient follow-up was performed by either a surgical, medical or radiation oncologist at the outpatient clinic of our hospital or affiliated hospitals. In general, an enhanced computed tomography (CT) of the chest, abdomen and pelvis was performed every three months within the first year postoperatively; after one year, an enhanced CT was performed every six months for another five years. If elevated CA19-9 levels were observed preoperatively, then this was re-evaluated every three months. Magnetic resonance imaging and/or ${ }^{18} \mathrm{~F}$-fluorodeoxyglucose positron emission tomography/CT were performed, if necessary, to clarify ambiguous CT findings.

When imaging findings were consistent with a recurrence, only the first site was documented. The recurrence sites were stratified into six mutually exclusive categories: liver, lung, peritoneum, remnant pancreas, local and other. Local recurrence was defined as recurrence within the surgical area such as the soft tissue along the aorta, superior mesenteric or celiac artery or around the hepaticojejunostomy or pancreatojejunostomy site. Other was defined as recurrence at other, less common sites. An ER was defined as a recurrence within the first 12 months after surgery in this study, as a previous study concluded that a recurrence-free interval of 12 months was the optimal threshold for differentiating between an ER and a late recurrence (LR) [6].

\subsection{Statistical Analyses}

The clinicopathological features were compared between patients who experienced a recurrence within the first year and those who did not. The categorical variables were compared using the $\chi^{2}$ test while the continuous variables were compared using a Student's $t$-test or the Mann-Whitney $U$ test. A receiver operating characteristic (ROC) curve was constructed to estimate the optimal cut-off value for the preoperative serum CA19-9 level as a risk factor for an ER; this was determined to be the point on the ROC curve closest to the upper-left corner of the graph. Associations between potential risk factors and an ER were assessed using a univariate logistic regression analysis. Variables with a $p$-value $<0.05$ were included as covariates in two separate multivariate logistic regression analyses: one for preoperative and one for postoperative risk factors associated with an ER.

The results are presented as odds ratios (ORs) with corresponding $95 \%$ confidence intervals (CIs). Overall survival (OS) was defined as the time from surgery to either death or the last follow-up. PRS was defined as the time from the first recurrence to either death or the last follow-up. Survival was calculated using the Kaplan-Meier method 
and compared between groups using the log-rank test; a two-sided $p$-value $<0.05$ was considered statistically significant. Statistical analyses were performed using BellCurve for Excel (version 3.21; Social Survey Research Information Co., Ltd., Tokyo, Japan).

\section{Results}

\subsection{Cohort Characteristics}

During the study period, a total of 234 patients comprising 121 men $(51.7 \%)$ and 113 women $(48.3 \%)$ underwent curative intent surgery for a newly diagnosed R- or BRPDAC. At the diagnosis, the cancer was R- in 171 patients $(73.1 \%)$, BR-portal vein (PV) in 43 patients $(18.4 \%)$ and BR-artery $(\mathrm{A})$ in 20 patients $(8.5 \%)$. The demographic, clinicopathological and treatment characteristics of the entire study population dichotomized by the presence or absence of recurrence are summarized in Table 1 . The average age \pm standard deviation (SD) was $69.3 \pm 9.0$ years. All patients underwent an oncological pancreatic resection. Depending on the location and extent of the tumor, a pancreatoduodenectomy $(n=155)$, distal pancreatectomy $(n=74)$ or total pancreatectomy $(n=5)$ was performed. Vascular resections were performed in 82 patients (35.0\%). Of the 82 patients, 70 underwent only vein resection, 10 underwent only artery resection and 2 underwent artery and vein resection combined. The tumor status following surgery was as follows: R0 in 220 patients $(94.0 \%), \mathrm{R} 1$ in 13 patients $(5.6 \%)$ and R2 in 1 patient $(0.6 \%)$. No significant differences were observed among the $\mathrm{R}$ status groups with respect to sex, the American Society of Anesthesiologist physical status (ASA PS) classification system, the body mass index (BMI), the surgical procedure, vascular resection, the histological type or the T-stage. The median preoperative serum CA19-9 level of 203 patients was $59.9 \mathrm{U} / \mathrm{mL}$. A ROC curve demonstrated that a preoperative serum CA19-9 value of $52 \mathrm{U} / \mathrm{mL}$ was the optimal cut-off point for an ER after surgery with a sensitivity and specificity of $72.5 \%$ and $55.5 \%$, respectively; the area under the ROC curve (AUC) was 0.6630.

Among all patients, 55 and 204 received neoadjuvant and adjuvant therapy, respectively. The median follow-up period was 25.2 (interquartile range, 13.8-43.4) months. At the last follow-up, a recurrence was documented for 141 patients $(60.3 \%)$; 93 patients $(39.7 \%)$ exhibited no signs of a recurrence after surgery. Significant differences between the recurrence and non-recurrence groups were observed in the age $(p=0.0165)$, resectability $(p=0.0116)$, preoperative CA19-9 level $(p<0.001)$, preoperative and pathological tumor size $(p=0.0018)$, positive lymph nodes $(p<0.001)$, microscopic perineural and lymphovascular invasion $(p<0.001)$, TNM stage $(p<0.001)$ and adjuvant therapy $(p=0.0012)$.

The median OS time for all patients was 38.5 months. The estimated 3- and 5-year survival rates were $50.2 \%$ and $37.3 \%$, respectively (Figure $1 \mathrm{~A}$ ). The median OS time in the recurrence group was 25.2 months; by comparison, survival did not reach the median time in the non-recurrence group with a significant difference observed between the groups $(p<0.001)$. The actual 3- and 5-year survival rates in the recurrence group were $30.0 \%$ and $13.3 \%$, respectively (Figure 1B). 
Table 1. Demographic, clinicopathological and treatment characteristics of included patients.

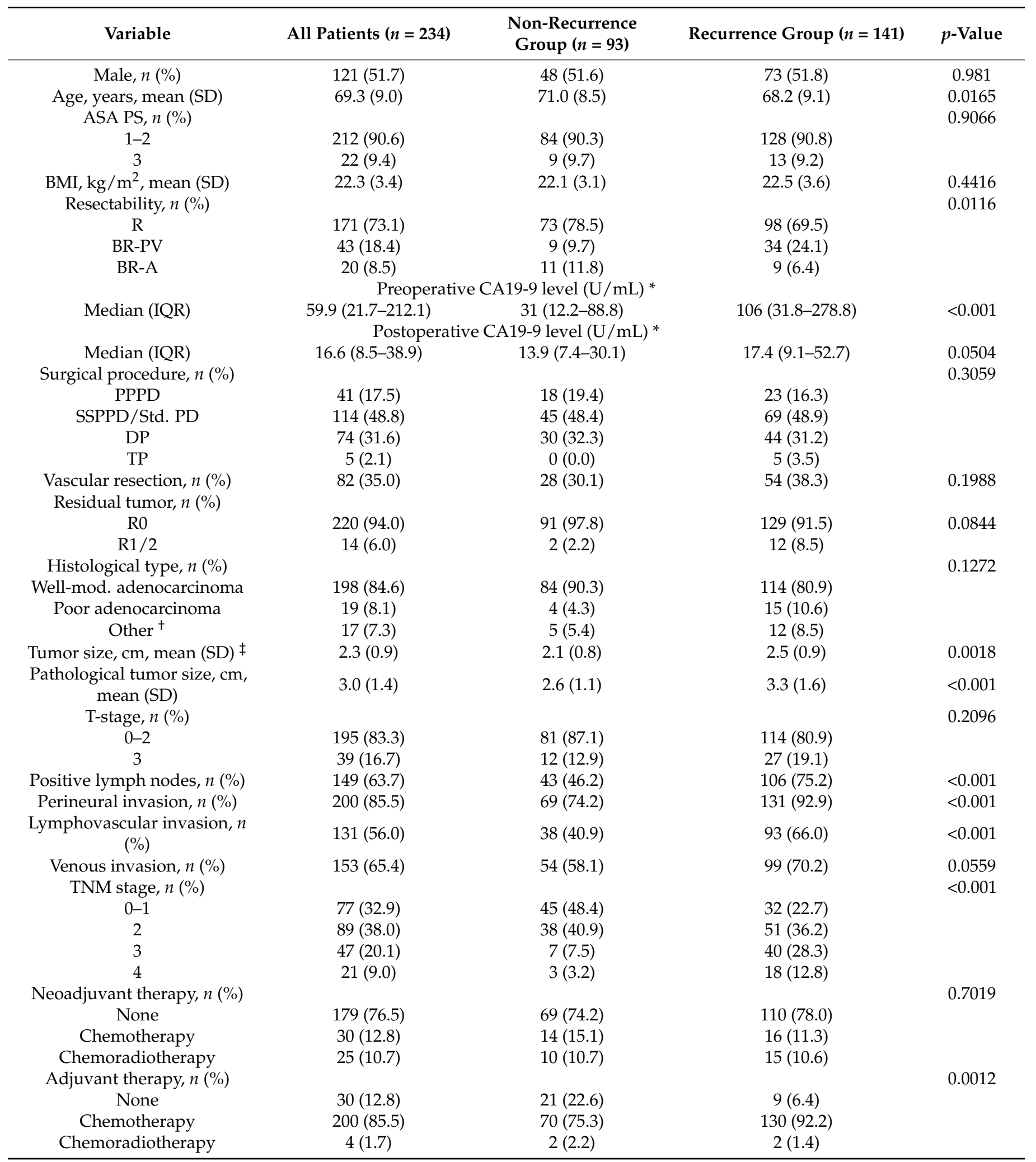

* Patients $(n=203)$ had perioperative CA19-9 measurements available. Patients $(n=31)$ with CA19-9 levels $<3 \mathrm{U} / \mathrm{mL}$ (related to Lewisnegative patients) were excluded. ${ }^{+}$Other consisted of histologic types such as adenosquamous $(n=10)$, anaplastic $(n=2)$, a high-grade pancreatic intraepithelial neoplasia $(n=2)$, a mixed neuroendocrine non-neuroendocrine neoplasm $(n=1)$ and an unclassifiable neoplasm $(n=2) .{ }^{\ddagger}$ Measured using endoscopic ultrasonography at the first examination and prior to neoadjuvant therapy. Abbreviations: ASA PS, American Society of Anesthesiologist physical status; BMI, body mass index; R, resectable; BR, borderline-resectable; PV, portal vein; A, artery; CA19-9, carbohydrate antigen 19-9; SD, standard deviation; IQR, interquartile range; mod., moderate; PPPD, pylorus-preserving pancreatoduodenectomy; SSPPD, subtotal stomach-preserving pancreatoduodenectomy; Std. PD, standard pancreatoduodenectomy; DP, distal pancreatectomy; TP, total pancreatectomy; mod., moderate; TNM, Tumor-Node-Metastasis. 


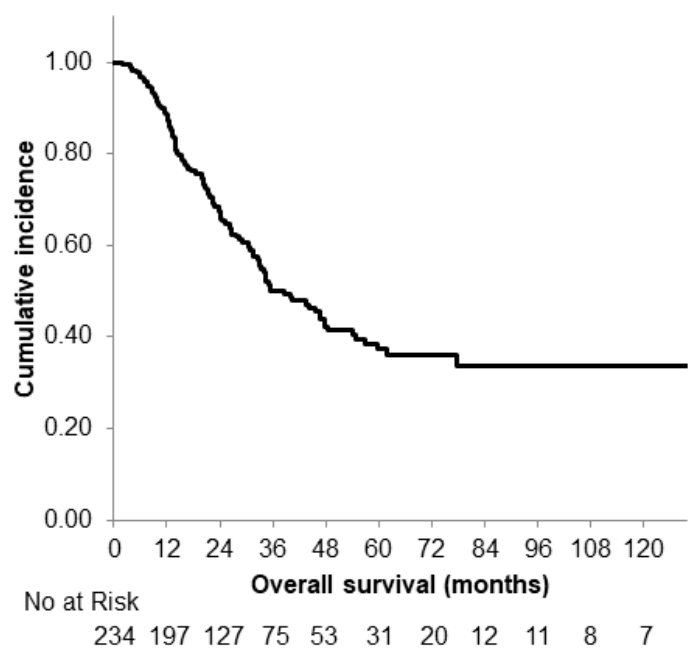

(A)

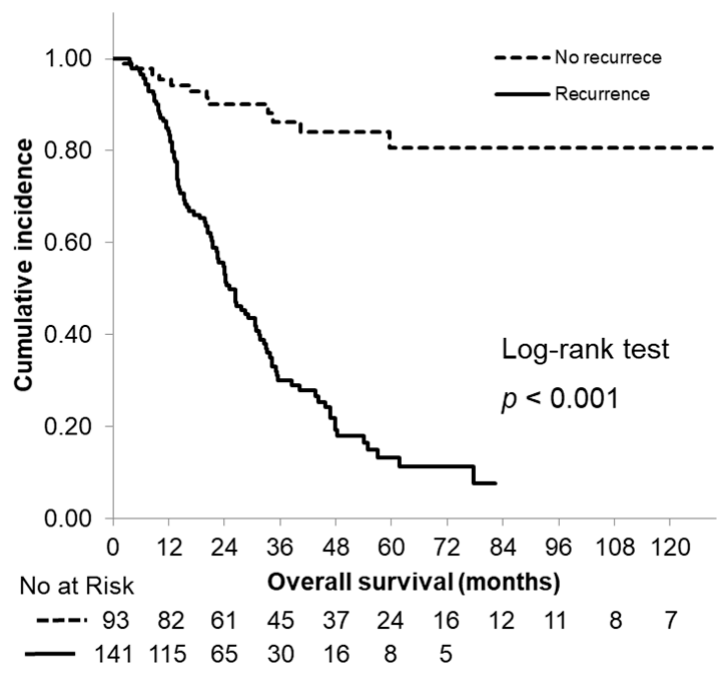

(B)

Figure 1. Kaplan-Meier curves of overall survival. (A) All patients; (B) patients stratified according to recurrence status.

\subsection{Postrecurrence Survival between Early and Late Recurrence}

Among the 141 patients with a recurrence after surgery, an ER was documented in 85 patients $(60.3 \%)$ and an LR in 56 patients (39.7\%). Patients with an ER tended to exhibit more poorly differentiated tumors as well as a microscopic lymphovascular invasion; additionally, postoperative CA19-9 levels were significantly higher (Table 2). Conversely, there were no significant differences in resectability, tumor size, TNM stage and neoadjuvant/adjuvant therapy between the ER and LR groups.

The median PRS time was significantly longer in patients with an LR (16.3 months, 95\% CI: 14.0-18.6) than in those with an ER (9.3 months, 95\% CI: 7.7-10.9) $(p<0.001$; Figure 2). The ER group had 1- and 2-year PRS rates of $38.7 \%$ and $9.5 \%$, compared with $66.9 \%$ and $37.2 \%$ in the LR group, respectively (both $p<0.001$ ).

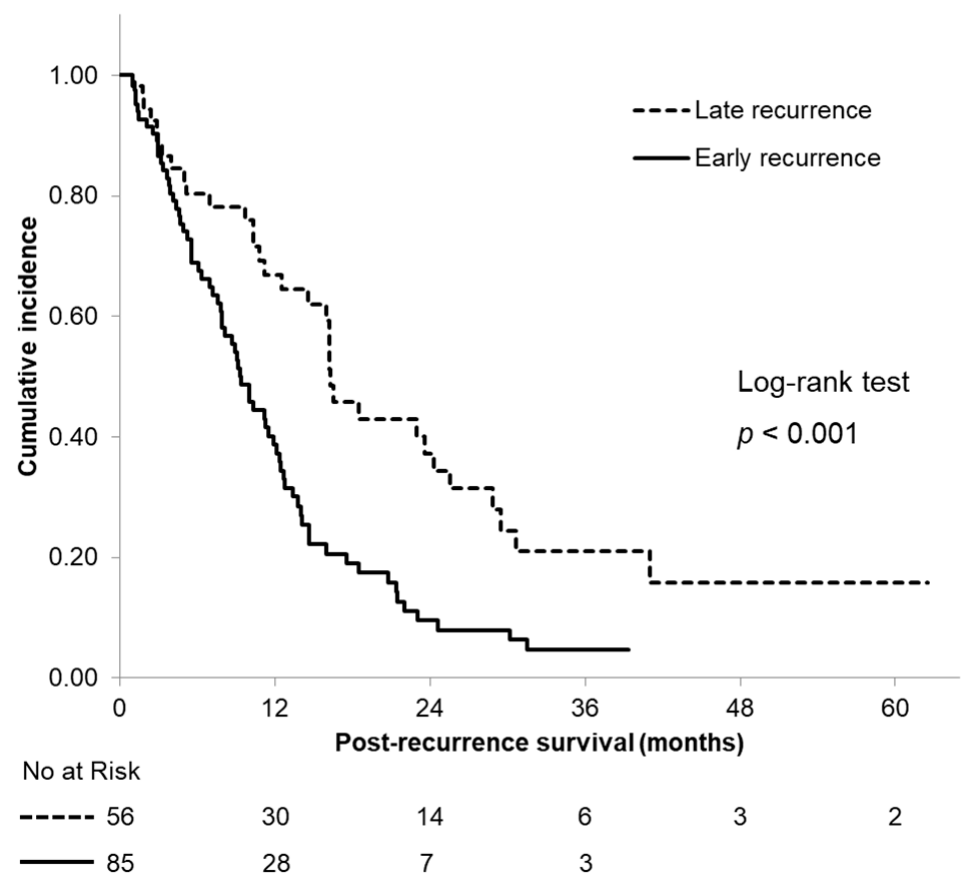

Figure 2. Kaplan-Meier curves of postrecurrence survival according to the time of recurrence. 
Table 2. Demographic, clinicopathological and treatment characteristics of patients with recurrence.

\begin{tabular}{|c|c|c|c|}
\hline Variable & ER Group $(n=85)$ & LR Group $(n=56)$ & $p$-Value \\
\hline Male, $n(\%)$ & $40(47.1)$ & $33(58.9)$ & 0.1675 \\
\hline Age, years, mean (SD) & $68.0(9.2)$ & $68.4(9.1)$ & 0.8119 \\
\hline ASA PS, $n(\%)$ & & & 0.1981 \\
\hline $1-2$ & $75(88.2)$ & $53(94.6)$ & \\
\hline 3 & $10(11.8)$ & $3(5.4)$ & \\
\hline BMI, $\mathrm{kg} / \mathrm{m}^{2}$, mean $(\mathrm{SD})$ & $22.4(3.7)$ & $22.6(3.4)$ & 0.8200 \\
\hline Resectability, $n(\%)$ & & & 0.7928 \\
\hline $\mathrm{R}$ & $60(70.6)$ & $38(67.9)$ & \\
\hline BR-PV & $19(22.4)$ & $15(26.8)$ & \\
\hline BR-A & $6(7.0)$ & $3(5.3)$ & \\
\hline \multicolumn{4}{|l|}{ Preoperative CA19-9 level (U/mL) * } \\
\hline Median (IQR) & $151.1(45.6-314.6)$ & $75.5(29.8-244.0)$ & 0.1733 \\
\hline \multicolumn{4}{|l|}{ Postoperative CA19-9 level $(\mathrm{U} / \mathrm{mL})$ * } \\
\hline Median (IQR) & $27.1(13.5-108.2)$ & $14.7(8-32)$ & 0.0113 \\
\hline Surgical procedure, $n(\%)$ & & & 0.1200 \\
\hline PPPD & $9(10.6)$ & $14(25.0)$ & \\
\hline SSPPD/Std. PD & $45(52.9)$ & $24(42.9)$ & \\
\hline $\mathrm{DP}$ & $27(31.8)$ & $17(30.3)$ & \\
\hline $\mathrm{TP}$ & $4(4.7)$ & $1(1.8)$ & \\
\hline Vascular resection, $n(\%)$ & $35(41.2)$ & $19(33.9)$ & 0.3863 \\
\hline Residual tumor, $n(\%)$ & & & 0.8852 \\
\hline R0 & $78(91.8)$ & $51(91.1)$ & \\
\hline $\mathrm{R} 1 / 2$ & $7(8.2)$ & $5(8.9)$ & \\
\hline Histological type, $n(\%)$ & & & 0.0360 \\
\hline Well-mod. Adenocarcinoma & $63(74.1)$ & $51(91.1)$ & \\
\hline Poor adenocarcinoma & $13(15.3)$ & $2(3.6)$ & \\
\hline Other $^{+}$ & $9(10.6)$ & $3(5.3)$ & \\
\hline Tumor size, cm, mean (SD) $\ddagger$ & $2.6(0.9)$ & $2.3(0.9)$ & 0.1876 \\
\hline $\begin{array}{l}\text { Pathological tumor size, } \mathrm{cm} \text {, mean } \\
\text { (SD) }\end{array}$ & $3.5(1.8)$ & $3.1(1.2)$ & 0.1572 \\
\hline T-stage, $n(\%)$ & & & 0.4509 \\
\hline $1-2$ & $67(78.8)$ & $47(83.9)$ & \\
\hline 3 & $18(21.2)$ & $9(16.1)$ & \\
\hline Positive lymph nodes, $n(\%)$ & $67(78.8)$ & $39(69.6)$ & 0.2169 \\
\hline Perineural invasion, $n(\%)$ & 79 (92.9) & $52(92.9)$ & 0.9848 \\
\hline Lymphovascular invasion, $n(\%)$ & $62(72.9)$ & $31(55.4)$ & 0.0311 \\
\hline Venous invasion, $n(\%)$ & $62(72.9)$ & $37(66.1)$ & 0.3828 \\
\hline TNM stage, $n(\%)$ & & & 0.3453 \\
\hline 1 & $17(20.0)$ & $15(26.8)$ & \\
\hline 2 & $29(34.1)$ & $22(39.3)$ & \\
\hline 3 & $25(29.4)$ & $15(26.8)$ & \\
\hline 4 & $14(16.5)$ & $4(7.1)$ & \\
\hline Neoadjuvant therapy, $n(\%)$ & & & 0.8380 \\
\hline None & $65(76.4)$ & $45(80.4)$ & \\
\hline Chemotherapy & $10(11.8)$ & $6(10.7)$ & \\
\hline Chemoradiotherapy & $10(11.8)$ & $5(8.9)$ & \\
\hline Adjuvant therapy, $n(\%)$ & & & 0.5223 \\
\hline None & $7(8.2)$ & $2(3.6)$ & \\
\hline Chemotherapy & $77(90.6)$ & $53(94.6)$ & \\
\hline Chemoradiotherapy & $1(1.2)$ & $1(1.8)$ & \\
\hline
\end{tabular}

* Patients $(n=121)$ had perioperative CA19-9 measurements available. Patients $(n=20)$ with CA19-9 levels $<3 \mathrm{U} / \mathrm{mL}$ (related to Lewis-negative patients) were excluded. ${ }^{\dagger}$ Other consisted of histologic types such as adenosquamous $(n=10)$, a mixed neuroendocrine non-neuroendocrine neoplasm $(n=1)$ and an unclassifiable neoplasm $(n=1)$. $\ddagger$ Measured using endoscopic ultrasonography at the first examination and prior to neoadjuvant therapy. Abbreviations: ER, early recurrence; LR, late recurrence; ASA PS, American Society of Anesthesiologist physical status; BMI, body mass index; R, resectable; BR, borderline-resectable; PV, portal vein; A, artery; CA19-9, carbohydrate antigen 19-9; SD, standard deviation; IQR, interquartile range; PPPD, pylorus-preserving pancreatoduodenectomy; SSPPD, subtotal stomach-preserving pancreatoduodenectomy; Std. PD, standard pancreatoduodenectomy; DP, distal pancreatectomy; TP, total pancreatectomy; mod., moderate; TNM, Tumor-Node-Metastasis. 


\subsection{Patterns of Early and Late Recurrence after Surgery}

The proportion and comparison of recurrence sites at different time points are shown in Figure 3A,B. The recurrence patterns were defined at the first recurrence location. These showed that in the ER group, 47 patients (55.3\%) initially exhibited a liver metastasis while 15 patients $(17.6 \%)$ initially exhibited a local recurrence. Conversely, in the LR group, 21 patients $(37.5 \%)$ had lung metastases while 11 patients $(19.6 \%)$ had a local recurrence. The ER group experienced significantly more frequent liver metastases than the LR group whereas the LR group experienced significantly more frequent lung metastases.

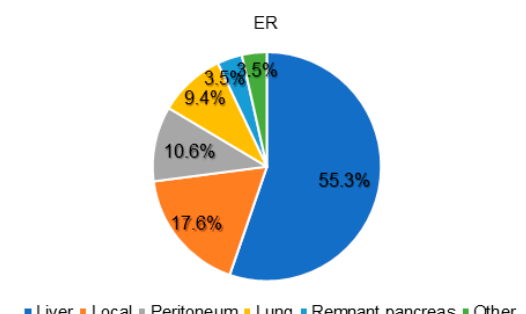

- Liver " Local | Peritoneum " Lung " Remnant pancreas = Other

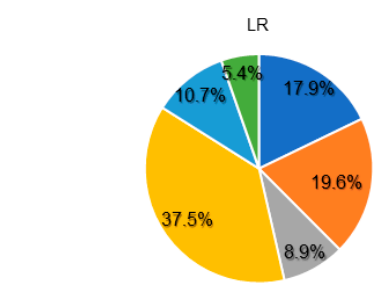

- Liver " Local || Peritoneum " Lung | Remnant pancreas | Other

\begin{tabular}{lccc}
\hline & $\operatorname{ER}(n=85)$ & $\operatorname{LR}(n=56)$ & $p$ Value \\
\hline Liver & $47(55.3 \%)$ & $10(17.9 \%)$ & $<0.001$ \\
Local & $15(17.6 \%)$ & $11(19.6 \%)$ & 0.765 \\
Peritoneum & $9(10.6 \%)$ & $5(8.9 \%)$ & 0.747 \\
Lung & $8(9.4 \%)$ & $21(37.5 \%)$ & $<0.001$ \\
Remnant pancreas & $3(3.5 \%)$ & $6(10.7 \%)$ & 0.088 \\
Other & $3(3.5 \%)$ & $3(5.4 \%)$ & 0.599 \\
\hline
\end{tabular}

(B)

Figure 3. (A) Proportion of first recurrence sites and (B) comparison of the recurrence patterns at different time points.

\subsection{Survival Analysis According to the Site of First Recurrence}

OS curves according to the site of the first recurrence are shown in Figure 4. Among all of the patients with a recurrence, 57 patients initially exhibited a liver metastasis, 29 patients initially exhibited a lung recurrence and 26 patients initially exhibited a local recurrence. The median OS time was 14.5 months in patients who initially exhibited a liver metastasis and 24.4 and 44.2 months in patients who initially exhibited a local recurrence and a lung metastasis, respectively; a significant difference was observed between the groups $(p<0.001)$.

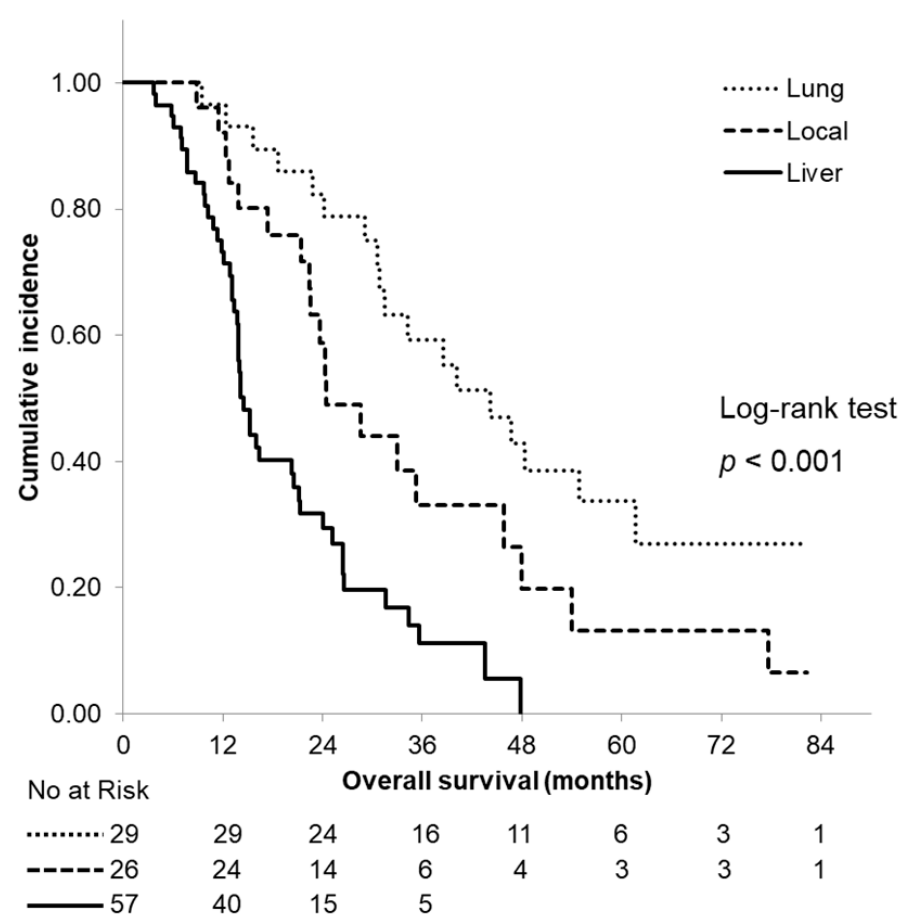

Figure 4. Kaplan-Meier curves of overall survival according to the site of the first recurrence. The median overall survival time was 14.5 months, 24.4 months and 44.2 months in patients who initially exhibited a liver metastasis, a local recurrence and a lung metastasis, respectively. 


\subsection{Risk Factors Associated with Early Recurrence}

The results of two separate univariate and multivariate logistic regression analyses of preoperative and postoperative risk factors are presented in Table 3. Two preoperative variables proved to be independently associated with a recurrence within 12 months after surgery: a preoperative tumor size $>3.0 \mathrm{~cm}$ on endoscopic ultrasonography at the first examination (OR: 3.11, 95\% CI: $1.35-7.14 ; p=0.0076$ ) and a preoperative CA19-9 level $>52 \mathrm{U} / \mathrm{mL}$ (OR: $3.25,95 \%$ CI: $1.67-6.30 ; p<0.001$ ). Two postoperative risk factors were independently correlated with an ER including a pathological tumor size $>3.0 \mathrm{~cm}$ (OR: 2.00, 95\% CI: 1.03-3.90; $p=0.0420$ ) and a postoperative CA19-9 level > $37 \mathrm{U} / \mathrm{mL}$ (OR: $2.11,95 \%$ CI: $1.02-4.36 ; p=0.0444)$. Conversely, both neoadjuvant and adjuvant therapy were not found to be independently associated with a reduction in an ER.

Table 3. Univariate and multivariate analyses of postoperative risk factors for an early recurrence after a resection.

\begin{tabular}{|c|c|c|c|c|}
\hline \multirow{2}{*}{ Preoperative Risk Factors } & \multicolumn{2}{|c|}{ Univariate } & \multicolumn{2}{|c|}{ Multivariate } \\
\hline & OR $(95 \% \mathrm{CI})$ & $p$-Value & OR $(95 \% \mathrm{CI})$ & $p$-Value \\
\hline Age (>70 vs. $\leq 70$ years) & $0.95(0.53-1.69)$ & 0.854 & - & - \\
\hline Sex (male vs. female) & $0.88(0.49-1.56)$ & 0.654 & - & - \\
\hline Resectability (BR vs. R) & $1.39(0.73-2.63)$ & 0.314 & - & - \\
\hline Tumor size $(>3.0$ vs. $\leq 3.0 \mathrm{~cm}) *$ & $3.05(1.37-6.77)$ & 0.0061 & $3.11(1.35-7.14)$ & 0.0076 \\
\hline Tumor location (head/uncinate vs. body/tail) & $1.01(0.54-1.88)$ & 0.983 & - & - \\
\hline Preoperative CA19-9 level (>52 vs. $\leq 52 \mathrm{U} / \mathrm{mL}$ ) & $3.30(1.76-6.19)$ & $<0.001$ & $3.25(1.67-6.30)$ & $<0.001$ \\
\hline Neoadjuvant therapy (yes vs. no) & $1.19(0.61-2.33)$ & 0.615 & - & - \\
\hline \multirow{2}{*}{ Postoperative Risk Factors } & \multicolumn{2}{|c|}{ Univariate } & \multicolumn{2}{|c|}{ Multivariate } \\
\hline & OR $(95 \%$ CI $)$ & $p$-Value & OR $(95 \% \mathrm{CI})$ & $p$-Value \\
\hline Age (>70 vs. $\leq 70$ years) & $0.95(0.53-1.69)$ & 0.854 & - & - \\
\hline Sex (male vs. female) & $0.88(0.49-1.56)$ & 0.654 & - & - \\
\hline Tumor size $(>3.0$ vs. $\leq 3.0 \mathrm{~cm})$ & $2.66(1.47-4.84)$ & 0.0013 & $2.00(1.03-3.90)$ & 0.0420 \\
\hline Tumor differentiation (poor vs. others) & $4.38(1.57-12.24)$ & 0.0049 & $2.32(0.75-7.22)$ & 0.1457 \\
\hline Positive lymph nodes (yes vs. no) & $2.61(1.36-5.02)$ & 0.0041 & $1.62(0.75-3.47)$ & 0.2184 \\
\hline Distant metastasis (yes vs. no) & $3.36(1.24-9.09)$ & 0.0173 & $1.66(0.55-4.98)$ & 0.3651 \\
\hline Perineural invasion (yes vs. no) & $3.16(1.16-8.63)$ & 0.0250 & $1.43(0.48-4.25)$ & 0.5213 \\
\hline Lymphovascular invasion (yes vs. no) & $2.86(1.54-5.32)$ & $<0.001$ & $1.74(0.85-3.56)$ & 0.1282 \\
\hline Venous invasion (yes vs. no) & $1.55(0.83-2.91)$ & 0.1665 & - & - \\
\hline Postoperative CA19-9 level (>37 vs. $\leq 37$ U /mL) & $3.18(1.63-6.23)$ & $<0.001$ & $2.11(1.02-4.36)$ & 0.0444 \\
\hline Adjuvant therapy (yes vs. no) & $2.19(0.78-6.13)$ & 0.137 & - & - \\
\hline
\end{tabular}

* Measured using endoscopic ultrasonography at the first examination and prior to neoadjuvant therapy. Abbreviations: CA19-9, carbohydrate antigen 19-9; CI, confidence interval; BR, borderline-resectable; OR, odds ratio; $\mathrm{R}$, resectable.

\section{Discussion}

The present study indicated that the median OS of patients with an R- and BR-PDAC was 38.5 months; $60.3 \%$ of these patients experienced a recurrence (both late and early) after curative surgery. The median OS of the recurrent cases was 25.2 months; the 5-year survival rate was $13.3 \%$. The proportion of patients with an ER after a curative resection for an R- and BR-PDAC was 36.3\%; the median PRS time was 9.3 months, which was significantly shorter than that of patients with an LR (16.3 months; $p<0.001)$. The most common site of an ER was the liver (55.3\%), which was observed more frequently than for an LR $(17.9 \% ; p<0.001)$. Moreover, there were significant differences in OS between a liver, local and lung recurrence; patients with a liver recurrence had a significantly shorter median OS time ( 14.5 months; $p<0.001)$. These results suggested that even if patients with an R- and BR-PDAC could be treated with curative surgery after neoadjuvant therapy, $>50 \%$ of them would experience a recurrence; additionally, their prognosis would be poor, especially in cases of an ER in the liver.

Recent studies [4,5] reported that even in the most favorable cohort (patients with an RPDAC), up to $80 \%$ of patients experienced a recurrence after a short recurrence-free interval. 
Another study [5] reported the liver to be the most common site of the first recurrence with a particularly low recurrence-free survival time with a median of 6.9 months. Nevertheless, there is some evidence to suggest that different sites of a recurrence have different survival rates [24-26]. Groot et al. [6] reported that patients with a liver recurrence had a worse survival than patients with a local or pulmonary recurrence. Interestingly, metachronous lung metastases as the first and only type of recurrence were found to develop later and had a better OS than presentations of metastatic disease [27,28]. These findings may suggest that patients with an ER in the liver had occult micrometastases that were undetectable using existing imaging modalities, suggesting the insufficiency of effective neoadjuvant therapies at the time of the resection.

In this study, several independent preoperative and postoperative factors associated with an increased likelihood of an ER after surgery for a PDAC were identified including preoperative and postoperative CA19-9 levels and tumor size. CA19-9 was first discovered in 1979 [29] and has become the most clinically useful and well-known biomarker for a PDAC. The CA19-9 level may be a reliable prognostic marker for survival, recurrence and tumor resectability [30,31]. Several reports have established an association between elevated preoperative and postoperative CA19-9 levels and a poor survival after a pancreatectomy for an R-PDAC, suggesting thresholds from 37 to $400 \mathrm{U} / \mathrm{mL}$ [32-35]. However, there are far fewer studies focusing on the correlation between the CA19-9 level and the ER of a PDAC (R- and BR-PDAC) and there is currently no consensus regarding the CA19-9 threshold for predicting an ER.

Groot et al. [6] reported that the optimal preoperative and postoperative CA19-9 cut-off values for predicting a recurrence within 12 months in patients with an R-PDAC $(n=957)$ were $>210$ and $>37 \mathrm{U} / \mathrm{mL}$, respectively. Nishio et al. [12] found that for an R-PDAC $(n=90)$, a preoperative CA19-9 level $>529 \mathrm{U} / \mathrm{mL}$ was an independent predictive factor for a recurrence within 12 months. Conversely, Tsai et al. [36] reported that following neoadjuvant therapy for an R- and BR-PDAC $(n=131)$, the normalization of CA19-9 levels was the strongest prognostic marker for a long-term survival.

In this study, the analysis of the ROC curve and the associated AUC values revealed that the optimal preoperative CA19-9 threshold for the prediction of an ER was $>52 \mathrm{U} / \mathrm{mL}$; however, with an AUC of 0.663 , sensitivity of $72 \%$ and specificity of $55 \%$, the predictive strength of an elevated preoperative CA19-9 level was fairly limited, highlighting the necessity of identifying more accurate biomarkers in patients with a PDAC.

Several methods targeting tumor associated molecules and genes have been investigated to detect an early postoperative recurrence of pancreatic cancer. The target specimens are divided into two main categories: preoperative and postoperative blood samples and resected tissues. Many research findings have been published regarding the association between tumor markers, blood counts and biochemical substances from peripheral blood and the early detection of a metastasis. Circulating nucleic acids and tumor cells are now considered to be additional targets for measurement. Circulating tumor cells (CTCs) are known to be important mediators for the development of metastases; their presence has been demonstrated in several malignancies including pancreatic, colorectal, gastric, ovarian, breast, prostate, bladder, renal and lung cancers [37]. The results of two large meta-analyses $[38,39]$ demonstrated a correlation between CTC positivity and poor outcomes in patients with pancreatic cancer; these analyses concluded that CTCs strongly predicted the disease course in patients with pancreatic cancer, indicating a poor OS and recurrence-free survival.

In a prospective longitudinal study (CLUSTER trial) including patients with pancreatic cancer [40], the number of CTCs decreased after neoadjuvant chemotherapy and surgery in patients with pancreatic cancer; patients who developed an ER within 12 months postoperatively had a significantly higher preoperative and postoperative CTC [41].

Studies using tumor tissues have reported an association between the ER of pancreatic cancer and the presence of cancer stem cells and novel substances found in omics studies. CD44 is one such marker of cancer stem cells and CD44-positive tumor cells were shown 
to be associated with tumor initiation, metastasis and prognosis [42]. In pancreatic cancer tissue, CD44 expression could therefore predict an ER [43].

Rho guanine nucleotide factor 2 (ARHGEF2, also known as GEF-H1) was extracted from a public database as the gene associated with an ER of pancreatic cancer [44]. In a mass spectrometry-based study [45], galectin 4, a group of carbohydrate-binding proteins involved in neoplastic development and progression, was identified as a downregulated protein in short-term survivors of pancreatic cancer and correlated with an ER [46]. Genome-wide DNA methylation screening revealed that three CpG marker sites including a CpG site in CDK14 could predict an ER in formalin-fixed, paraffin-embedded surgically resected tissue [47].

The results of this study suggested that large tumor diameters or high CA19-9 levels, even after neoadjuvant therapy, may lead to an ER after a pancreatectomy. However, existing clinical parameters alone are insufficient to predict an ER after a pancreatectomy and it is necessary to apply the so-called "liquid biopsy" such as an analysis of CTCs to clinical trials to predict occult micrometastases. The accurate preoperative identification of patients with a high likelihood of an ER will be beneficial for the patients and help the clinicians in decision-making regarding the extension of the neoadjuvant therapy duration as well as the choice of adjuvant therapy for a PDAC.

This study has several limitations worthy of consideration. First, this was a retrospective study conducted at a single institution with a relatively small sample size. As is the case with retrospective studies, the present study consisted of accurate and sufficient data to perform the analysis with adequate precision; however, there were a few missing elements that could not be retrospectively analyzed. Second, approximately $5-10 \%$ of the general population were Lewis antigen A- and B-negative, meaning they were unable to synthesize the CA19-9 antigen and could therefore not exhibit elevated CA19-9 levels even in cases of pancreatic cancer. Finally, neoadjuvant therapy includes a variety of regimens reflecting the adaptation of the patients to clinical trials at the time and this variation may have resulted in a degree of error due to a variance in the analysis.

\section{Conclusions}

This study found that preoperatively $(>52 \mathrm{U} / \mathrm{mL}$ ) and postoperatively $(>37 \mathrm{U} / \mathrm{mL})$ elevated CA19-9 levels as well as a tumor size $>3.0 \mathrm{~cm}$ were independently associated with an ER after a pancreatectomy for both an R- and BR-PDAC. Furthermore, an ER resulted in a more frequent liver metastasis than an LR, suggesting that patients experiencing a recurrence within 12 months had undetected micrometastases. Further studies are needed to identify new biomarkers for the detection of a clinically occult micrometastatic disease at the time of operation as the currently acknowledged preoperative risk factors are inadequate to accurately identify patients susceptible to an ER of a PDAC.

Author Contributions: Conceptualization: M.I., M.N. and Y.K.; methodology: M.I. and M.N.; formal analysis: M.I. and D.K.; collection and/or assembly of data: S.O., T.M., A.K. and T.K.; data analysis and interpretation: M.I., D.K. and H.Y.; writing—original draft preparation: M.I.; writing—review and editing: M.I., M.N., D.K., H.Y., T.K., Y.K. and I.T.; visualization: M.I. and A.K. All authors have read and agreed to the published version of the manuscript.

Funding: This research received no external funding.

Institutional Review Board Statement: The study was conducted in accordance with the Declaration of Helsinki. The retrospective study design was approved by the Institutional Review Board of Sapporo Medical University Hospital (Sapporo, Japan) (approval number: 292-68).

Informed Consent Statement: The need for patient consent was waived because this was an observational study that used available samples and information; the data did not include individual identification codes. In addition, the opportunity for patients to opt out was always available on the hospital website.

Data Availability Statement: Data sharing is not applicable to this article. The authors presented all the necessary information in the manuscript. 
Conflicts of Interest: The authors declare no conflict of interest.

\section{References}

1. Rahib, L.; Smith, B.D.; Aizenberg, R.; Rosenzweig, A.B.; Fleshman, J.M.; Matrisian, L.M. Projecting Cancer Incidence and Deaths to 2030: The Unexpected Burden of Thyroid, Liver, and Pancreas Cancers in the United States. Cancer Res. 2014, 74, 2913-2921. [CrossRef]

2. Hori, M.; Matsuda, T.; Shibata, A.; Katanoda, K.; Sobue, T.; Nishimoto, H.; Japan Cancer Surveillance Research Group. Cancer Incidence and Incidence Rates in Japan in 2009: A Study of 32 Population-Based Cancer Registries for the Monitoring of Cancer Incidence in Japan (MCIJ) Project. Jpn. J. Clin. Oncol. 2015, 45, 884-891. [CrossRef] [PubMed]

3. Tempero, M.A.; Malafa, M.P.; Chiorean, E.G.; Czito, B.; Scaife, C.; Narang, A.K.; Fountzilas, C.; Wolpin, B.M.; Al-Hawary, M.; Asbun, H.; et al. Pancreatic adenocarcinoma, version 1.2019 featured updates to the NCCN guidelines. J. Natl. Compr. Cancer Netw. 2019, 17, 203-210.

4. $\quad$ Suenaga, M.; Fujii, T.; Kanda, M.; Takami, H.; Okumura, N.; Inokawa, Y.; Kobayashi, D.; Tanaka, C.; Yamada, S.; Sugimoto, H.; et al. Pattern of First Recurrent Lesions in Pancreatic Cancer: Hepatic Relapse Is Associated with Dismal Prognosis and Portal Vein Invasion. Hepato-Gastroenterology 2014, 61, 1756-1761. [PubMed]

5. Groot, V.P.; Rezaee, N.; Wu, W.; Cameron, J.L.; Fishman, E.K.; Hruban, R.H.; Weiss, M.J.; Zheng, L.; Wolfgang, C.L.; He, J. Patterns, Timing, and Predictors of Recurrence Following Pancreatectomy for Pancreatic Ductal Adenocarcinoma. Ann. Surg. 2018, 267, 936-945. [CrossRef] [PubMed]

6. Groot, V.P.; Gemenetzis, G.; Blair, A.B.; Rivero-Soto, R.J.; Yu, J.; Javed, A.A.; Burkhart, R.A.; Rinkes, I.H.M.B.; Molenaar, I.Q.; Cameron, J.L.; et al. Defining and predicting early recurrence in 957 patients with Resected Pancreatic Ductal Adenocarcinoma. Ann. Surg. 2019, 269, 1154-1162. [CrossRef]

7. La Torre, M.; Nigri, G.; Lo Conte, A.; Mazzuca, F.; Tierno, S.M.; Salaj, A.; Marchetti, P.; Ziparo, V.; Ramacciato, G. Is a Preoperative Assessment of the Early Recurrence of Pancreatic Cancer Possible After Complete Surgical Resection? Gut Liver 2014, 8 , 102-108. [CrossRef]

8. Kim, N.; Han, I.W.; Ryu, Y.; Hwang, D.W.; Heo, J.S.; Choi, D.W.; Shin, S.H. Predictive Nomogram for Early Recurrence After Pancreatectomy in Resectable Pancreatic Cancer: Risk Classification Using Preoperative Clinicopathologic Factors. Cancers 2020, 12, 137. [CrossRef]

9. Sugiura, T.; Uesaka, K.; Kanemoto, H.; Mizuno, T.; Sasaki, K.; Furukawa, H.; Matsunaga, K.; Maeda, A. Serum CA19-9 Is a Significant Predictor Among Preoperative Parameters for Early Recurrence After Resection of Pancreatic Adenocarcinoma. J. Gastrointest. Surg. 2012, 16, 977-985. [CrossRef]

10. Waraya, M.; Yamashita, K.; Katagiri, H.; Ishii, K.; Takahashi, Y.; Furuta, K.; Watanabe, M. Preoperative Serum CA19-9 and Dissected Peripancreatic Tissue Margin as Determiners of Long-Term Survival in Pancreatic Cancer. Ann. Surg. Oncol. 2009, 16, 1231-1240. [CrossRef]

11. Van Manen, L.; Groen, J.V.; Putter, H.; Pichler, M.; Vahrmeijer, A.L.; Bonsing, B.A.; Mieog, J.S.D. Stage-Specific Value of Carbohydrate Antigen 19-9 and Carcinoembryonic Antigen Serum Levels on Survival and Recurrence in Pancreatic Cancer: A Single Center Study and Meta-Analysis. Cancers 2020, 12, 2970. [CrossRef]

12. Nishio, K.; Kimura, K.; Amano, R.; Yamazoe, S.; Ohrira, G.; Nakata, B.; Hirakawa, K.; Ohira, M. Preoperative Predictors for Early Recurrence of Resectable Pancreatic Cancer. World J. Surg. Oncol. 2017, 15, 16. [CrossRef]

13. Barugola, G.; Partelli, S.; Marcucci, S.; Sartori, N.; Capelli, P.; Bassi, C.; Pederzoli, P.; Falconi, M. Resectable Pancreatic Cancer: Who Really Benefits from Resection? Ann. Surg. Oncol. 2009, 16, 3316-3322. [CrossRef]

14. La Torre, M.; Nigri, G.; Cavallini, M.; Mercantini, P.; Ziparo, V.; Ramacciato, G. The Glasgow Prognostic Score as a Predictor of Survival in Patients with Potentially Resectable Pancreatic Adenocarcinoma. Ann. Surg. Oncol. 2012, 19, 2917-2923. [CrossRef]

15. Kurahara, H.; Maemura, K.; Mataki, Y.; Sakoda, M.; Iino, S.; Kawasaki, Y.; Arigami, T.; Mori, S.; Kijima, Y.; Ueno, S.; et al. A Therapeutic Strategy for Resectable Pancreatic Cancer Based on Risk Factors of Early Recurrence. Pancreas 2018, 47, 753-758. [CrossRef]

16. Jang, J.Y.; Han, Y.; Lee, H.; Kim, S.W.; Kwon, W.; Lee, K.H.; Oh, D.Y.; Chie, E.K.; Lee, J.M.; Heo, J.S.; et al. Oncological Benefits of Neoadjuvant Chemoradiation With Gemcitabine Versus Upfront Surgery in Patients with Borderline Resectable Pancreatic Cancer: A Prospective, Randomized, Open-Label, Multicenter Phase 2/3 Trial. Ann. Surg. 2018, 268, 215-222. [CrossRef]

17. Chawla, A.; Molina, G.; Pak, L.M.; Rosenthal, M.; Mancias, J.D.; Clancy, T.E.; Wolpin, B.M.; Wang, J. Neoadjuvant Therapy Is Associated with Improved Survival in Borderline-Resectable Pancreatic Cancer. Ann. Surg. Oncol. 2020, 27, 1191-1200. [CrossRef] [PubMed]

18. Unno, M.; Motoi, F.; Matsuyama, Y.; Satoi, S.; Matsumoto, I.; Aosasa, S.; Shirakawa, H.; Wada, K.; Fujii, T.; Yoshitomi, H.; et al. Randomized Phase II/III Trial of Neoadjuvant Chemotherapy with Gemcitabine and S-1 Versus Upfront Surgery for Resectable Pancreatic Cancer (Prep-02/JSAP-05). J. Clin. Oncol. 2019, 37, 189. [CrossRef]

19. Parra-Robert, M.; Santos, V.M.; Canis, S.M.; Pla, X.F.; Fradera, J.M.A.; Porto, R.M. Relationship Between CA 19.9 and the Lewis Phenotype: Options to Improve Diagnostic Efficiency. Anticancer Res. 2018, 38, 5883-5888. [CrossRef] [PubMed]

20. Das, R.; McGrath, K.; Seiser, N.; Smith, K.; Uttam, S.; Brand, R.E.; Fasanella, K.E.; Khalid, A.; Chennat, J.S.; Sarkaria, S.; et al. Tumor Size Differences Between Preoperative Endoscopic Ultrasound and Postoperative Pathology for Neoadjuvant-Treated Pancreatic Ductal Adenocarcinoma Predict Patient Outcome. Clin. Gastroenterol. Hepatol. 2020. [CrossRef] [PubMed] 
21. Brierley, J.D.; Gospodarowicz, M.K.; Wittekind, C. TNM Classification of Malignant Tumours, 8th ed.; Wiley Blackwell: New York, NY, USA, 2017; ISBN 9781119263579.

22. Oettle, H.; Post, S.; Neuhaus, P.; Gellert, K.; Langrehr, J.; Ridwelski, K.; Schramm, H.; Fahlke, J.; Zuelke, C.; Burkart, C.; et al. Adjuvant Chemotherapy with Gemcitabine vs Observation in Patients Undergoing Curative-Intent Resection of Pancreatic Cancer: A Randomized Controlled Trial. JAMA 2007, 297, 267-277. [CrossRef]

23. Uesaka, K.; Boku, N.; Fukutomi, A.; Okamura, Y.; Konishi, M.; Matsumoto, I.; Kaneoka, Y.; Shimizu, Y.; Nakamori, S.; Sakamoto, H.; et al. JASPAC 01 Study Group. Adjuvant Chemotherapy of S-1 Versus Gemcitabine for Resected Pancreatic Cancer: A phase 3, Open-Label, Randomised, Non-Inferiority Trial (JASPAC 01). Lancet 2016, 388, 248-257. [CrossRef]

24. Hishinuma, S.; Ogata, Y.; Tomikawa, M.; Ozawa, I.; Hirabayashi, K.; Igarashi, S. Patterns of Recurrence After Curative Resection of Pancreatic Cancer, Based on Autopsy Findings. J. Gastrointest. Surg. 2006, 10, 511-518. [CrossRef]

25. Sperti, C.; Pasquali, C.; Piccoli, A.; Pedrazzoli, S. Recurrence After Resection for Ductal Adenocarcinoma of the Pancreas. World J. Surg. 1997, 21, 195-200. [CrossRef]

26. Groot, V.P.; Gemenetzis, G.; Blair, A.B.; Ding, D.; Javed, A.A.; Burkhart, R.A.; Yu, J.; Borel Rinkes, I.H.; Molenaar, I.Q.; Cameron, J.L.; et al. Implications of the Pattern of Disease Recurrence on Survival Following Pancreatectomy for Pancreatic Ductal Adenocarcinoma. Ann. Surg. Oncol. 2018, 25, 2475-2483. [CrossRef] [PubMed]

27. Downs-Canner, S.; Zenati, M.; Boone, B.A.; Varley, P.R.; Steve, J.; Hogg, M.E.; Zureikat, A.; Zeh, H.J.; Lee, K.K. The Indolent Nature of Pulmonary Metastases from Ductal Adenocarcinoma of the Pancreas. J. Surg. Oncol. 2015, 112, 80-85. [CrossRef] [PubMed]

28. Lovecek, M.; Skalicky, P.; Chudacek, J.; Szkorupa, M.; Svebisova, H.; Lemstrova, R.; Ehrmann, J.; Melichar, B.; Yogeswara, T.; Klos, D.; et al. Different Clinical Presentations of Metachronous Pulmonary Metastases After Resection of Pancreatic Ductal Adenocarcinoma: Retrospective Study and Review of the Literature. World J. Gastroenterol. 2017, 23, 6420-6428. [CrossRef]

29. Koprowski, H.; Steplewski, Z.; Mitchell, K.; Herlyn, M.; Herlyn, D.; Fuhrer, P. Colorectal Carcinoma Antigens Detected by Hybridoma Antibodies. Somat. Cell Genet. 1979, 5, 957-971. [CrossRef]

30. Papalezova, K.T.; Tyler, D.S.; Blazer, D.G.; Clary, B.M.; Czito, B.G.; Hurwitz, H.I.; Uronis, H.E.; Pappas, T.N.; Willett, C.G.; White, R.R. Does Preoperative Therapy Optimize Outcomes in Patients with Resectable Pancreatic Cancer? J. Surg. Oncol. 2012, 106, 111-118. [CrossRef] [PubMed]

31. Blazer, M.; Wu, C.; Goldberg, R.M.; Phillips, G.; Schmidt, C.; Muscarella, P.; Wuthrick, E.; Williams, T.M.; Reardon, J.; Ellison, E.C.; et al. Neoadjuvant Modified (m) FOLFIRINOX for Locally Advanced Unresectable (LAPC) and Borderline Resectable (BRPC) Adenocarcinoma of the Pancreas. Ann. Surg. Oncol. 2015, 22, 1153-1159. [CrossRef] [PubMed]

32. Berger, A.C.; Garcia, M.; Hoffman, J.P.; Regine, W.F.; Abrams, R.A.; Safran, H.; Konski, A.; Benson, A.B., III; MacDonald, J.; Willett, C.G. Postresection CA 19-9 Predicts Overall Survival in Patients with Pancreatic Cancer Treated With Adjuvant Chemoradiation: A Prospective Validation by RTOG 9704. J. Clin. Oncol. 2008, 26, 5918. [CrossRef]

33. Reni, M.; Cereda, S.; Balzano, G.; Passoni, P.; Rognone, A.; Fugazza, C.; Mazza, E.; Zerbi, A.; Di Carlo, V.; Villa, E. Carbohydrate Antigen 19-9 Change During Chemotherapy for Advanced Pancreatic Adenocarcinoma. Cancer 2009, 115, 2630-2639. [CrossRef]

34. Turrini, O.; Schmidt, C.M.; Moreno, J.; Parikh, P.; Matos, J.M.; House, M.G.; Zyromski, N.J.; Nakeeb, A.; Pitt, H.A.; Lillemoe, K.D. Very High Serum CA 19-9 Levels: A Contraindication to Pancreaticoduodenectomy? J. Gastrointest. Surg. 2009, 13, 1791-1797. [CrossRef]

35. Barton, J.G.; Bois, J.P.; Sarr, M.G.; Wood, C.M.; Qin, R.; Thomsen, K.M.; Kendrick, M.L.; Farnell, M.B. Predictive and Prognostic Value of CA 19-9 in Resected Pancreatic Adenocarcinoma. J. Gastrointest. Surg. 2009, 13, 2050-2058. [CrossRef]

36. Tsai, S.; George, B.; Wittmann, D.; Ritch, P.S.; Krepline, A.N.; Aldakkak, M.; Barnes, C.A.; Christians, K.K.; Dua, K.; Griffin, M.; et al. Importance of Normalization of CA19-9 Levels following Neoadjuvant Therapy in Patients with Localized Pancreatic Cancer. Ann. Surg. 2020, 271, 740-747. [CrossRef]

37. Allard, W.J.; Matera, J.; Miller, M.C.; Repollet, M.; Connelly, M.C.; Rao, C.; Tibbe, A.G.; Uhr, J.W.; Terstappen, L.W. Tumor Cells Circulate in the Peripheral Blood of All Major Carcinomas but Not in Healthy Subjects or Patients with Nonmalignant Diseases. Clin. Cancer Res. 2004, 10, 6897-6904. [CrossRef] [PubMed]

38. Ma, X.L.; Li, Y.Y.; Zhang, J.; Huang, J.W.; Jia, H.Y.; Liu, L.; Li, P. Prognostic Role of Circulating Tumor Cells in Patients with Pancreatic Cancer: A Meta-Analysis. Asian Pac. J. Cancer Prev. 2014, 15, 6015-6020. [CrossRef]

39. Han, L.; Chen, W.; Zhao, Q. Prognostic Value of Circulating Tumor Cells in Patients with Pancreatic Cancer: A Meta-Analysis. Tumour Biol. 2014, 35, 2473-2480. [CrossRef] [PubMed]

40. Poruk, K.E.; Valero, V.; Saunders, T.; Blackford, A.L.; Griffin, J.F.; Poling, J.; Hruban, R.H.; Anders, R.A.; Herman, J.; Zheng, L.; et al. Circulating Tumor Cell Phenotype Predicts Recurrence and Survival in Pancreatic Adenocarcinoma. Ann. Surg. 2016, 264, 1073-1081. [CrossRef]

41. Gemenetzis, G.; Groot, V.P.; Yu, J.; Ding, D.; Teinor, J.A.; Javed, A.A.; Wood, L.D.; Burkhart, R.A.; Cameron, J.L.; Makary, M.A.; et al. Circulating Tumor Cells Dynamics in Pancreatic Adenocarcinoma Correlate with Disease Status: Results of the Prospective CLUSTER Study. Ann. Surg. 2018, 268, 408-420. [CrossRef]

42. Zöller, M. CD44: Can a Cancer-Initiating Cell Profit from an Abundantly Expressed Molecule? Nat. Rev. Cancer 2011, 11, 254-267. [CrossRef]

43. Hsu, C.P.; Lee, L.Y.; Hsu, J.T.; Hsu, Y.P.; Wu, Y.T.; Wang, S.Y.; Yeh, C.N.; Chen, T.C.; Hwang, T.L. CD44 Predicts Early Recurrence in Pancreatic Cancer Patients Undergoing Radical Surgery. Vivo 2018, 32, 1533-1540. [CrossRef] [PubMed] 
44. Nakao, Y.; Nakagawa, S.; Yamashita, Y.I.; Umezaki, N.; Okamoto, Y.; Ogata, Y.; Yasuda-Yoshihara, N.; Itoyama, R.; Yusa, T.; Yamashita, K.; et al. High ARHGEF2 (GEF-H1) Expression is Associated with Poor Prognosis Via Cell Cycle Regulation in Patients with Pancreatic Cancer. Ann. Surg. Oncol. 2021. [CrossRef] [PubMed]

45. Hu, D.; Ansari, D.; Pawłowski, K.; Zhou, Q.; Sasor, A.; Welinder, C.; Kristl, T.; Bauden, M.; Rezeli, M.; Jiang, Y.; et al. Proteomic Analyses Identify Prognostic Biomarkers for Pancreatic Ductal Adenocarcinoma. Oncotarget 2018, 9, 9789-9807. [CrossRef]

46. Hu, D.; Ansari, D.; Zhou, Q.; Sasor, A.; Said Hilmersson, K.; Andersson, R. Galectin 4 Is a Biomarker for Early Recurrence and Death After Surgical Resection for Pancreatic Ductal Adenocarcinoma. Scand. J. Gastroenterol. 2019, 54, 95-100. [CrossRef]

47. Endo, Y.; Fujimoto, M.; Ito, N.; Takahashi, Y.; Kitago, M.; Gotoh, M.; Hiraoka, N.; Yoshida, T.; Kitagawa, Y.; Kanai, Y.; et al. Clinicopathological Impacts of DNA Methylation Alterations on Pancreatic Ductal Adenocarcinoma: Prediction of Early Recurrence Based on Genome-Wide DNA Methylation Profiling. J. Cancer Res. Clin. Oncol. 2021, 147, 1341-1354. [CrossRef] [PubMed] 